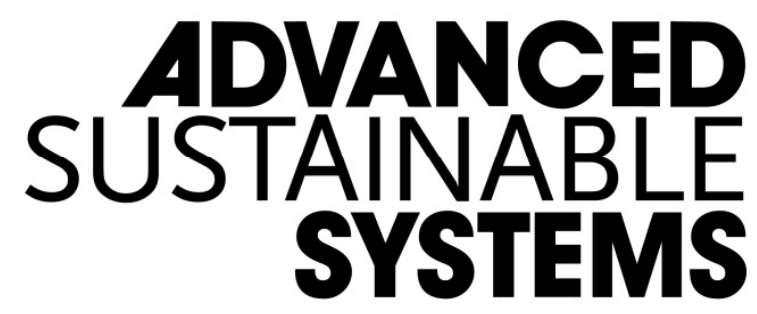

Dear Author,

Please correct your galley proofs carefully and return them no more than four days after the page proofs have been received.

Please limit corrections to errors already in the text; cost incurred for any further changes or additions will be charged to the author, unless such changes have been agreed upon by the editor.

The editors reserve the right to publish your article without your corrections if the proofs do not arrive in time.

Note that the author is liable for damages arising from incorrect statements, including misprints.

Please note any queries that require your attention. These are indicated with a $Q$ in the PDF and a question at the end of the document.

Reprints may be ordered by filling out the accompanying form.

Return the reprint order form by fax or by e-mail with the corrected proofs, to WileyVCH : advsustainsys@wiley.com
Postfach 101161 69451 Weinheim

Germany

Courier services:

Boschstraße 12

69469 Weinheim

Germany

Tel.: (+49) 6201606531

Fax: (+49) 6201606500

E-mail: advsustainsys@wiley.com

\title{
WILEY-VCH
}

To avoid commonly occurring errors, please ensure that the following important items are correct in your proofs (please note that once your article is published online, no further corrections can be made):

- Names of all authors present and spelled correctly

- Titles of authors correct (Prof. or Dr. only: please note, Prof. Dr. is not used in the journals)

- Addresses and postcodes correct

- E-mail address of corresponding author correct (current email address)

- Funding bodies included and grant numbers accurate

- Title of article OK

- All figures included

- Equations correct (symbols and sub/superscripts)

Corrections should be made directly in the PDF file using the PDF annotation tools. If you have questions about this, please contact the editorial office. The corrected PDF and any accompanying files should be uploaded to the journal's Editorial Manager site. 


\section{FULL PAPERS}

$x \mathrm{xxx}$

S. Goswami, S. Nandy,* J. Deuermeier, A. C. Marques, D. Nunes, S. P. Patole, P. M. F. J. Costa, R. Martins,

E. Fortunato* 1700137

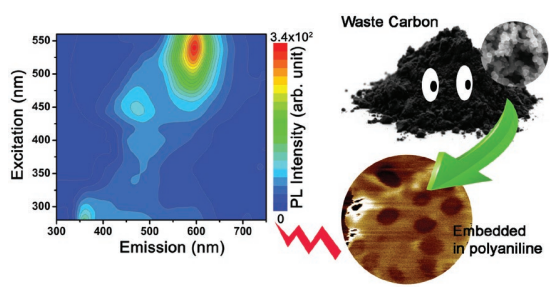

Cooking oven produced carbon is in- 1 corporated into polyaniline without any 3 further purification or chemical modifica- 4 tion. The product shows excitation-inde- 5 pendent triple-band photoluminescence, 6 as well as sensitive and selective ion de- 7

Green Nanotechnology from Waste tection $\left(\mathrm{Fe}^{+3}\right)$ ability. Clearly, it would add 8 up to the green nanotechnology area, 9 Carbon-Polyaniline Composite: Generation of Wavelength-Independent beneficiary to the society.

Multiband Photoluminescence for Sensitive Ion Detection 


\title{
Green Nanotechnology from Waste Carbon-Polyaniline Composite: Generation of Wavelength-Independent Multiband Photoluminescence for Sensitive Ion Detection
}

\author{
Sumita Goswami, Suman Nandy,* Jonas Deuermeier, Ana C. Marques, Daniela Nunes, \\ Shashikant P. Patole, Pedro M. F. J. Costa, Rodrigo Martins, and Elvira Fortunato*
}

This study reports on the qualitative analysis of photoluminescence effect generated from waste carbon of cooking oven by facile cost-effective material engineering. The waste carbon product as a form of carbon nanoparticles (CNPs) is incorporated within a conjugate polymer, namely, polyaniline (PANI) to produce CNP-PANI composites that have shown excitationwavelength-independent triple-band photoluminescence emission effect and highly sensitive $\mathrm{Fe}^{+3}$ ion detection ability. Herein the waste carbon material, while functionalized within the conjugated polymer, needs no further acid treatment or surface modification thus making the process cheaper, environmentally benign, and useful for green nanotechnology. The excitationwavelength-independent unique triple-band photoluminescence spectrum is the direct consequence of carbon-polyaniline synergy in $\pi-\pi$ transition and the surface passivation of CNPs by the $-\mathrm{NH}_{2}$ group rich aniline during in $\Omega$ situ polymerization. The current scenario has been studied for the samples prepared with different CNP concentrations for different reaction times and discussed in details with supportive physico-chemical characterizations. Moreover, the present study has demonstrated that the current material can be used as a fluorescent sensing platform for $\mathrm{Fe}^{+3}$ ions with high sensitivity and selectivity criteria whan the detection limit of the sensing probe has a value as low as $12 \times 10$

\section{Introduction}

With global interest, carbon-based nanomaterials having significant implications for the development of optoelectronic

\footnotetext{
S. Goswami, S. Nandy, J. Deuermeier, A. C. Marques, D. Nunes,

R. Martins, E. Fortunato

i3N/CENIMAT

Department of Materials Science

Faculty of Science and Technology

Universidade NOVA de Lisboa and CEMOP/UNINOVA

Campus de Caparica 2829-516 Caparica, Portugal

E-mail: snandy_ju@yahoo.co.in, s.nandy@fct.unl.pt; emf@fct.unl.pt

S. P. Patole, P. M. F. J. Costa

Physical Science and Engineering Division

King Abdullah University of Science and Technology

Thuwal 23955-6900, Saudi Arabia

The ORCID identification number(s) for the author(s) of this article can be found under https://doi.org/10.1002/adsu.201700137.
}

DOI: $10.1002 /$ adsu.201700137
13

devices, ${ }^{[1-3]}$ energy conversion, ${ }^{[4]}$ photo- 14 catalysis, ${ }^{[5]}$ biomedical imaging, ${ }^{[6-8]} 15$ sensing probe, ${ }^{[9-14]}$ and many more, ${ }^{[15,16]} 16$ are receiving great attention from both 17 experimentalists and theorists. They 18 have become the new-generation lumi- 19 nescent materials being superior to 20 conventional organic dyes and semicon- 21 ductor quantum dots, while compared in 22 terms of chemical inertness, large two- 23 photon excitation cross sections, lack of 24 blinking, low cytotoxicity, and biocompat- 25 ibility. ${ }^{[17]}$ However, proper understanding 26 of the photoluminescence (PL) origins in 27 carbon nanoparticles is still inadequate, 28 which is actually lowering the speed of 29 major development and abundant use of 30 carbon-based materials with desired PL 31 characteristics. Different mechanisms 32 have been proposed so far, respectable 33 ones are, for example, quantum confine- 34 ment effect or conjugated $\pi$-domains 35 determined by carbon core, surface state 36 determined by hybridization of carbon 37 backbone and connected chemical 38 groups, molecule state determined by 39 solely fluorescent molecules connected 40 on surface or inside the carbon particles as well as the cross- 41 link enhanced emission effect. Though the mechanisms 42 based on particle size distribution, surface traps and quantum 43 effects, charge transfer, intramolecular H-bonds, electronic 44 conjugate structure, defects, etc., are more popular in this 45 case, till now, the actual PL mechanism is still an open debate 46 for related researchers. ${ }^{[18-22]}$ On the other side, conjugate 47 polymers are also of great interest because of their ability to 48 control the energy gap and electronegativity through molec- 49 ular design that has made possible the synthesis of con- 50 ducting polymers with a range of ionization potentials and 51 electron affinities. ${ }^{[23,24]}$ More interestingly, most of the con- 52 jugated polymer shows PL quenching upon oxidation, while 53 developing oxidation-resistance can lead to an increment of 54 PL emission. The excellent properties of $\pi$-electron delocali- 55 zation at the backbone of conjugate polymer can be an initi- 56 ator of charge transfer mechanism with carbon nanoparticles 57 (CNPs) resulting oxidation-resistance. This unique combina- 58 tion of two types of materials has the ability to expand the 59 
1 class of novel composite materials with controlling their prop2 erties through rational chemical synthesis. ${ }^{[2,26]}$

In this work, we have used the idea of surface passivation indirectly by the incorporation of CNPs within polyaniline (PANI) during in situ polymerization. CNPs that were collected from normal cooking oven produced waste carbon, feature significantly simpler acquisition and low cost, but have rarely been studied as photoluminescence material. A simple and feasible wet chemical route has been followed using ethanol-water as 10 the reaction and dispersion medium. Aniline monomer acted 11 as the key factor for polymerization and in a late run also pro12 viding the surface amino groups for passivating surface traps.

13 As-synthesized product has shown highly intense triple-band 14 photoluminescent peaks, interestingly with excitation-wave15 length-independent behavior. Different concentrations of CNPs 16 have been incorporated into the conjugated polymer, driving 17 to the development of oxidation-resistant polymers that show 18 a significant outcome on PL emission. On the other hand, a 19 progressive study on the PL emission with functionalization 20 time of CNPs within PANI was carried out to understand 21 the influence of interaction between them. Our findings can 22 be considered among the few reports, which have achieved the 23 excitation-independent luminescence in carbon-based materials. 24 Though both excitation-dependent and excitation-independent 25 emissions have been observed, the excitation wavelength 26 dependent emission behavior is most common and it may 27 arise from the wide distributions of differently sized dots and 28 surface chemistry, different emissive traps, or some other cur29 rently unresolved source. ${ }^{[27]}$ But, the excitation-independent 30 PL behavior has been seldom achieved and the reason behind 31 it is still unclear. Researchers are mostly doing their part for 32 improving quantum yield of CNPs, ${ }^{[28,29]}$ though widening the 33 area of comprehensive understanding of emission origins and 34 finding out the controlling factors or criteria are also very much 35 significant for more successful applications in future. Even 36 after all these yet to be clear concepts about the PL of CNPs, 37 several research groups have accepted the idea of explaining 38 their radiative transition through the effective role of surface 39 traps. In most of the situations, the excitation dependence of 40 CNPs could be controlled through managing the emissive trap41 ping states introduced by surface groups such as $\mathrm{C}-\mathrm{O}, \mathrm{C}=\mathrm{O}$, $42 \mathrm{O}=\mathrm{C}-\mathrm{OH}$, etc., with various energy levels. But, if all the sur43 face states of CNPs are passivated, the emission is supposed produced waste carbon as the source of CNPs which can be a great replacement for the expensive and unsustainable source materials thus stepping toward the green nanotechnology.

\section{Results and Discussions}

\subsection{Surface and Chemical Structure Analyses}

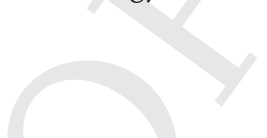

Detailed information about the chemical composition of as-synthesized CNP-incorporated PANI samples has been explored using tapping mode phase imaging atomic force microscopy (AFM) (PI-AFM) technique by applying sample bias voltage. PI-AFM is a sensitive nanoscale probing method to detect the phase shift of cantilever oscillation and its proximal interaction with sample surface, associated with stiffness, viscoelasticity, and chemical composition of the material. ${ }^{[32]}$ Here, we have applied surface voltage during phase imaging of sample so that it can impart enhancement of the image contrast with effect of localized electrostatic field. Figure 1a shows the PI of heterogeneous regions in phase-separated CNP-incorporated PANI composite. Circular spots with average size of $40 \mathrm{~nm}$ can be ascribed to CNPs within polymer matrix. Observed topographical features of the corresponding area (Figure 1b) do not show any kind of structural effects, which corroborates that PI-AFM is the result of a localized variation of different chemical compositional effects. Such PI is quite effective to determine the surface modification of polymer materials containing nanoparticles. Figure S1a,b (Supporting Information) is representative field emission scanning electron microscopy (FESEM) images of carbon nanoparticles (as directly obtained from the waste carbon product of cooking oven) and the typical CNP-incorporated PANI samples, respectively.

The X-ray photoelectron spectroscopy (XPS) results indicate that a typical CNP-PANI sample is mainly composed of carbon, nitrogen, and oxygen (see Figure S3, Supporting Information). In order to speculate on the influence of incorporated CNPs on PANI, C1s and N1s core levels are further quantitatively deconvoluted which is shown in Figure 2a. The high-resolution spectrum of $\mathrm{C} 1 \mathrm{~s}$ exhibits four main peaks, centered at about $284.3(\mathrm{C}-\mathrm{C}$ or $\mathrm{C}-\mathrm{H}), 285.0(\mathrm{C}=\mathrm{N}), 286.7\left(\mathrm{C}=\mathrm{N}^{+}\right.$or $\left.-\mathrm{C}-\mathrm{O}\right)$, and $288.6(>\mathrm{C}=\mathrm{O})$ eV. ${ }^{[33-35]}$ The typical XPS N1s core-level spectra for CNP-PANI sample are shown in Figure 2a. The relatively broad peak suggests the existence of different component peaks. Thus, the high-resolution peak of N1s is deconvoluted and reasonably fitted with four Gaussian-Lorentzian peaks having binding energy values of $\approx 398.1,399.2,400.7$, and $402.2 \mathrm{eV}$. The peak with the lowest binding energy $(398.1 \mathrm{eV})$ is due to imine-like $(=\mathrm{N}-$ ) structure, and the peak centered at $399.2 \mathrm{eV}$ is attributed to amine-like nitrogen atoms $(-\mathrm{NH}-) \cdot{ }^{[33,36]}$ The peak centered at 400.7 is attributed to cationic nitrogen atoms on the polymer backbone and the highest binding energy peak $(402.2 \mathrm{eV})$ is due to the protonated amine units. ${ }^{[33,36]}$ The XPS data were compared to a pure PANI sample as well as to samples of varying amount of CNPs (not shown here). No systematic changes in $\mathrm{C} 1 \mathrm{~s}$ and $\mathrm{N} 1 \mathrm{~s}$ could be detected, which is an indication that CNPs are embedded in PANI and do not reside on the surface, confirming the AFM measurement. Fourier transformed infrared (FTIR) and Raman spectroscopy

\section{1} 2 3 4 5 6 7 

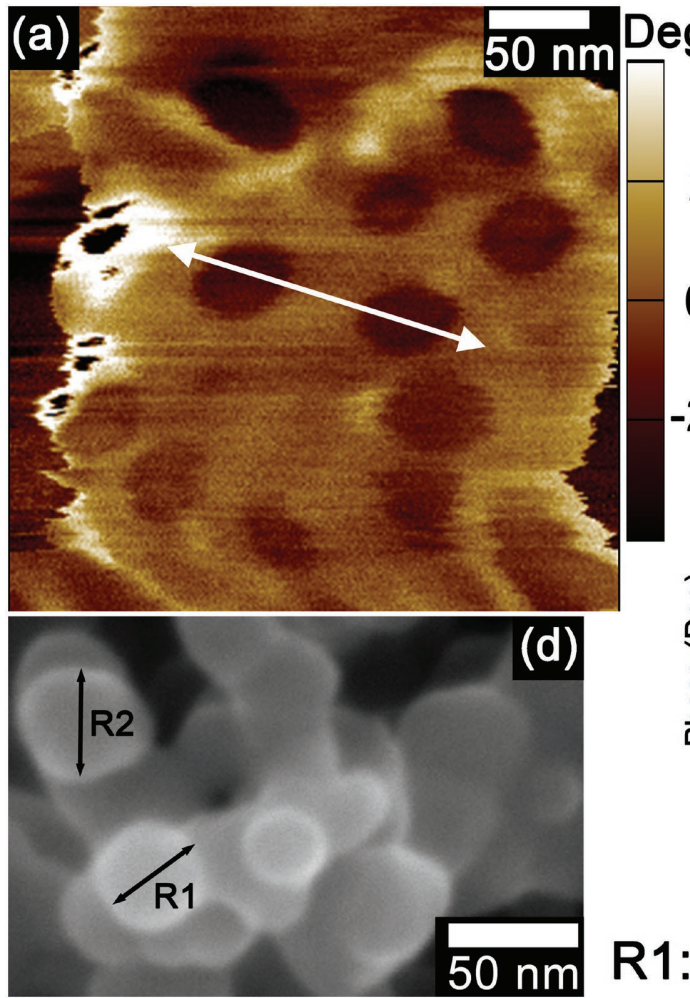

Figure 1. Localized phase imaging map of CNP-PANI. a) Localized phase imaging map of a heterogeneous region of polyaniline surface containing carbon nanoparticles with the bias voltage of $2 \mathrm{~V}$. Image contrast with dark balls indicates the presence of CNPs. b) The topographical features of the same area which shows nothing informative on chemical compositional effects. c) Corresponding profile (white arrow in PI) has been displayed which indicates an average of 38-40 nm carbon particle in size. d) A SEM image of carbon particles with average size of $40 \mathrm{~nm}$ has been shown which is well matched with PI profile.

were used to analyze the chemical structure of as-prepared samples. A FTIR spectrum of typical CNP-PANI sample with different synthesis time duration is depicted in Figure $2 \mathrm{~b}$. As expected, some typical characteristic absorption bands of PANI were observed in the spectra. For instance, 1560-1558 and $1488-1456 \mathrm{~cm}^{-1}$ (due to stretching vibration of $\mathrm{C}=\mathrm{C}$ in quinoid (Q) and benzenoid (B) rings, respectively), 1305-1285 $\mathrm{cm}^{-1}$ (attributed to $\pi$-electron delocalization induced in polymer by protonation of doping process), 1251-1233 $\mathrm{cm}^{-1}$ (associated with $\mathrm{C}-\mathrm{N}$ stretching of the secondary aromatic amine), $1152-1110 \mathrm{~cm}^{-1}$ (due to aromatic $\mathrm{C}-\mathrm{H}$ in-plane bending), as well as $820-814 \mathrm{~cm}^{-1}$ (related to out-of-plane deformation of $\mathrm{C}-\mathrm{H}$ in the 1,4-disubstitued benzene ring) were observed. ${ }^{[37]}$ Thus, FTIR spectra display essentially the same absorptive characteristics as that of pristine PANI. ${ }^{[38,39]}$ But, there is slight red shifting in peak positions with different reaction time durations which may be attributed to the incorporation of CNPs. The quinoid/benzenoid (Q/B) ratio when calculated from FTIR spectra for a particular sample is observed to decrease with increasing time duration of polymerization, that is, with preceding functionalization of CNPs within the polymer. Also, the Q/B ratio as determined by FTIR has smaller values for the sample prepared using higher CNP concentrations (Figure S4, Supporting Information) signifying the presence of more reduced emeraldine segments in these functionalized materials. This can be

\section{R1: $38.5 \mathrm{~nm}, \mathrm{R} 2: 42.1 \mathrm{~nm}$}
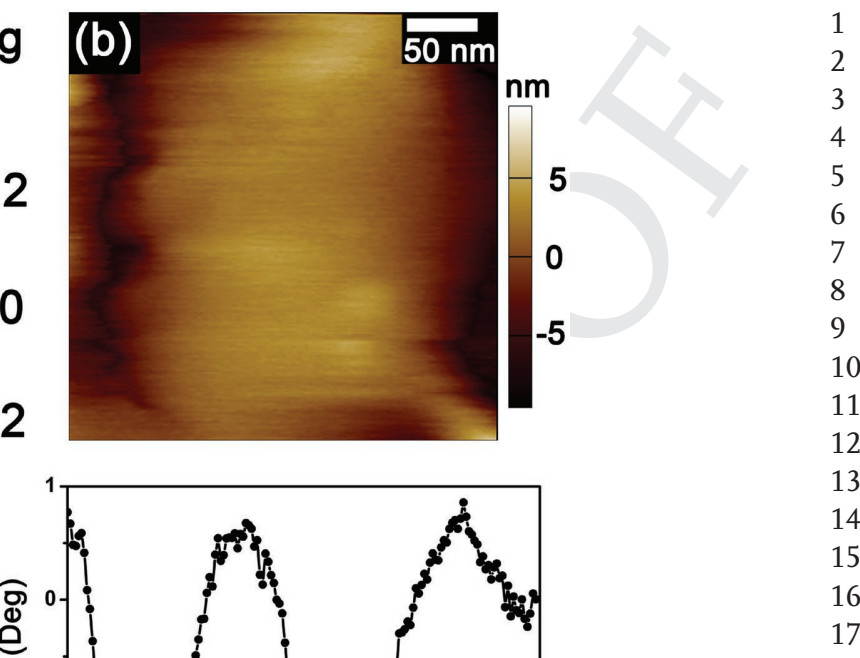
! ! ! ? !
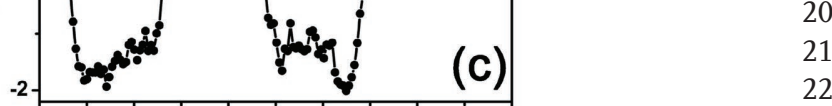

23

24 25 26

attributed to strong interactions existing between CNPs and 35 PANI in which the functionalized CNPs reduce PANI through 36 charge transfer.

Figure 3 shows the Raman spectra of a typical CNP-PANI 38 sample (CP, for time T1-T4) excited by $532 \mathrm{~nm}$ laser line in 39 air ambient environment. In the spectra, two very intense but 40 broad and diffused peaks can be seen as main characteris- 41 tics. So, they are deconvoluted into the fitted ones to obtain 42 a relevant idea about constituent elements, that is, CNPs 43 and PANI. The vibrational bands at $\approx 1356$ and $1564 \mathrm{~cm}^{-1} 44$ are the so-called D and $G$ bands of carbon for disorder- 45 induced feature (usually due to the presence of amorphous 46 disordered carbon structure of CNPs) and stretching mode 47 of $\mathrm{C}-\mathrm{C}$ bonds, respectively. ${ }^{[40]}$ Here, the intensity ratio of $\mathrm{D} 48$ and G-band $\left(I_{\mathrm{D}} / I_{\mathrm{G}}\right)$ is found to have decreasing value due to 49 decrease in the relative intensity of D-band with increasing 50 polymerization and CNP incorporation. It indicates that the 51 effective defects are decreasing which is also further supported 52 by PL results showing narrow-sharp peak with increasing 53 time for a particular sample. On the other hand, the con- 54 ventional Raman spectra of PANI have bands situated at the 55 regions: $1165-1520 \mathrm{~cm}^{-1}$ (different $\mathrm{C}-\mathrm{N}$ stretching modes- 56 amines, imines, polarons, bipolarons) and $1570-1650 \mathrm{~cm}^{-1} 57$ (quinoid or benzenoid rings $\mathrm{C}-\mathrm{C}$ stretching modes). ${ }^{[41,42]}$ In 58 the present spectral decomposition, the contributory peaks 59 
(a)

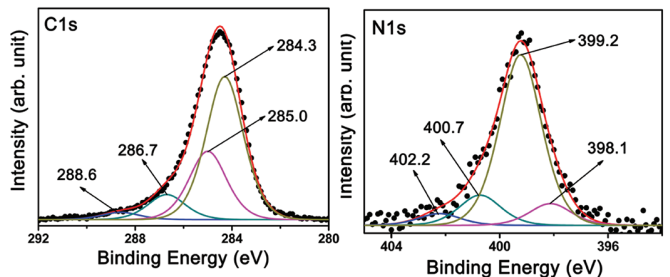

(b)

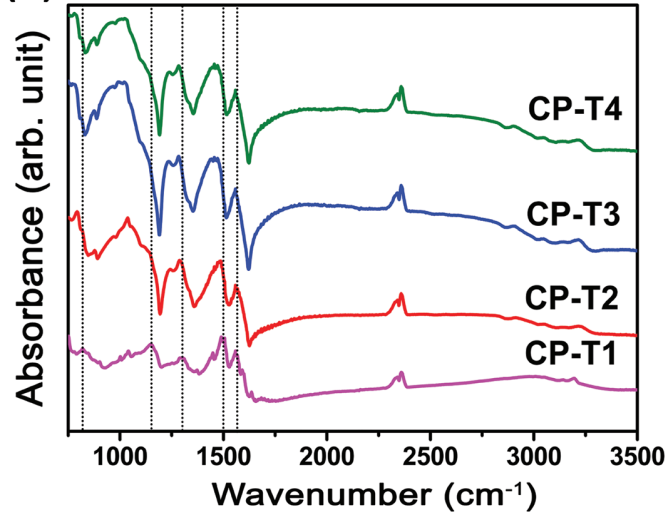

Figure 2. Analytical spectra of XPS and FTIR. a) Selective high-resolution XPS spectra of $\mathrm{Cls}$ and N1s. b) Analytical FTIR spectra of sample series of $\mathrm{CP}$ for different reaction time $\mathrm{T} 1, \mathrm{~T} 2, \mathrm{~T} 3$, and $\mathrm{T} 4(1.5,2,2.5$, and $3 \mathrm{~h}$, respectively). The dotted lines in the figure indicate the blue shift of some peaks which can be associated with reaction time of CNP-PANI.
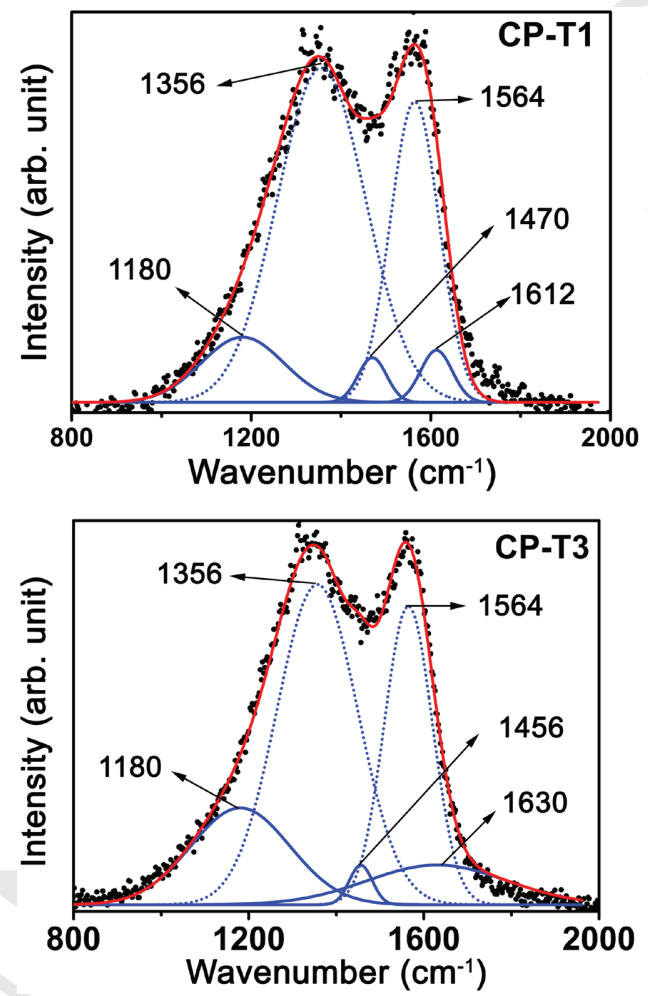

from PANI are at around 1180, 1456-1470, and 1612-1649 $\mathrm{cm}^{-1} \quad 1$ (some peaks cannot be even decomposed for PANI due to the 2 immense effect of CNPs in D and G-band positions, there- 3 fore neglected for analysis). Relative intensity of the band 4 at around $1180 \mathrm{~cm}^{-1}$ has been increased with increasing 5 polymerization time (T1-T4), verifying the predominance 6 of polaronic carriers from benzenoid rings. The band at 7 $1470 \mathrm{~cm}^{-1}$ has decreasing intensity with time and shifts toward 8 lower wavenumber region down to $1456 \mathrm{~cm}^{-1}$. This can be 9 attributed to $\mathrm{C}=\mathrm{N}$ stretching of the quinoid and the shifting 10 is due to incorporation and attachment of CNPs within PANI. 11 The peak around $1612-1649 \mathrm{~cm}^{-1}$ is due to $\mathrm{C}-\mathrm{C}$ stretching 12 effects from quinoid and benzenoid rings but difficult to sepa- 13 rate for verifying their individual contribution. This subpeak 14 (as shown in Figure 3a-d) becomes very broad (FWHM value 15 increases from 83.0 to 387.0 ) with increasing polymerization 16 time and subsequently has also its center shifted from 1612 to 17 $1649 \mathrm{~cm}^{-1}$ for corresponding samples CP-T1 to CP-T4. Such kind of increase in FWHM is already reported by Salvatierra et al. ${ }^{[42]}$ and is in the benzenoid part of the peak. Hence in 20 this work here, the particular Raman peak broadening with 21 increasing time from T1 to T4 indicates the formation of 22 PANI enriched with more benzenoid units, when the amount 23 of CNPs in the nanocomposites is higher. It also further gives 24 the reason behind enhanced PL performance. Therefore, both 25 Raman and FTIR indicate the functionalization of CNPs by 26 PANI to form CNP-PANI where the amino group has played 27 a key role for surface passivation, thus generating enhanced 28
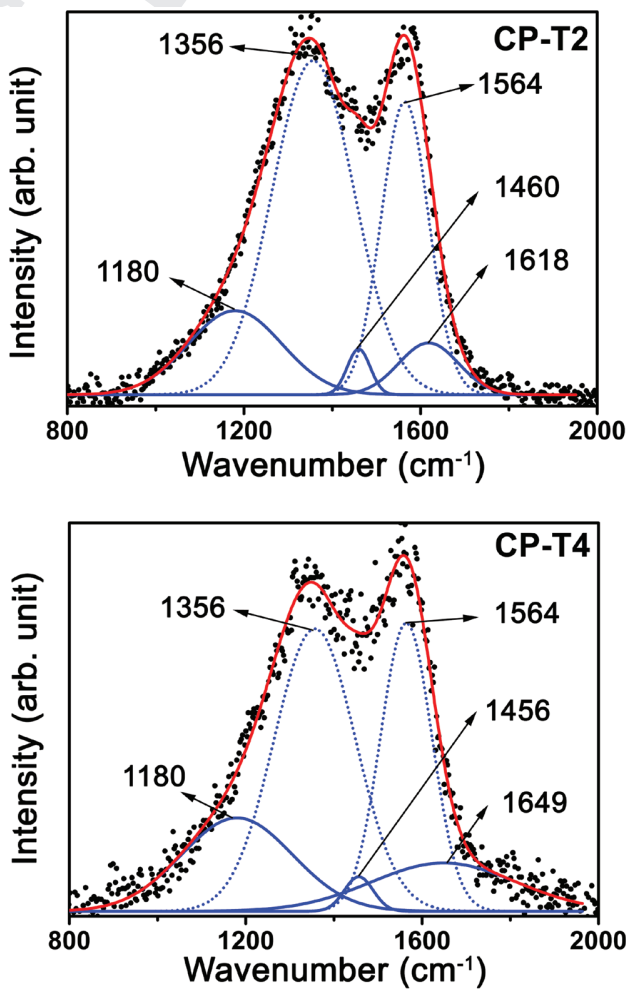

Figure 3. Raman spectra of CNP-PANI. Fitted Raman bands of sample series of CP for different reaction times T1, T2, T3, and T4. The red line indicates the fitted total profile adjustment where black dots have displayed the experimental data. Dotted blue lines are fitted for D and G bands of amorphous CNPs incorporated in PANI. The peak ration of $I_{D} / I_{G}$ decreases with the polymerization time (T1 to T4). Solid blue lines are fitted for three peak regions from PANI. . , .

.
(1)

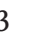
14 1

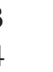

3


photoluminescence properties of CNPs and PANI qualitatively and quantitatively.

\subsection{Optical Behavior of CNP-PANI}

To investigate the optical properties of as-prepared CNP-PANI samples, UV-vis absorbance and PL spectra of the CNP-PANI dispersions were analyzed. Figure 4a shows several characteristic absorption peaks of CNP-PANI composites. The absorption bands, observed at 230 and $268 \mathrm{~nm}$ can be assigned to $\pi-\pi^{*}$ (aromatic $\mathrm{sp}^{2}$ domains) and $\mathrm{n}-\pi^{*}$ (broken symmetry transition from $\mathrm{C}=\mathrm{O}$ ) transitions in functionalized CNPs. ${ }^{[43,44]}$ Other absorption around $368 \mathrm{~nm}$ corresponds to $\pi-\pi^{*}$ benzenoid transition within PANI. ${ }^{[40,45]}$ Besides, the connected chemical groups between CNPs and PANI may contribute to the absorption region band starting from 320 to $560 \mathrm{~nm}$, which can arise from $\pi-\pi$ interaction. Absorption at 368 and $460 \mathrm{~nm}$ can be attributed to a transition from $\sigma$ and $\pi$ orbital (HOMO) to the lowest unoccupied molecular orbital (LUMO).
A circumstantial study of PL emission has been performed 1 upon using this energy band as excitation.

CNP-PANI nanocomposites in the present study have exhib- 3 ited excitation-independent triple-band emission which is an 4 excellent outcome to utilize the waste carbon product for fur- 5 ther optoelectronic applications at the same time making the 6 process economic and green. A dynamic study of PL character- 7 istics has been performed for the assorted series of composites 8 for different amour $\mathrm{f}$ carbon nanoparticles functionalized 9 within polyaniline ( $\mathrm{CP}$, and h-CP series) associating with 10 different reaction times (T1-T4). The particular of samples with 11 their notation have been given in the Experimental Section. 12 Figure 4b represents triple-band PL emission spectra for 13 the sample series of CP for different functionalization time 14 when excited at $280 \mathrm{~nm}$. The strong emission peak at $360 \mathrm{~nm} 15$ $\left(I_{\mathrm{P} 1}\right)$ may be attributed to benzenoid $\pi-\pi^{*}$ transition in the 16 polymer backbone due to the well-matched absorption band 17 information obtained earlier during the UV-vis study (i.e., the 18 peak at $368 \mathrm{~nm}$ corresponding to benzenoid $\pi-\pi *$ transition of 19 PANI component). ${ }^{[46]}$ The other peak at $\approx 466 \mathrm{~nm}\left(I_{\mathrm{P} 2}\right)$ is due 20 (a)
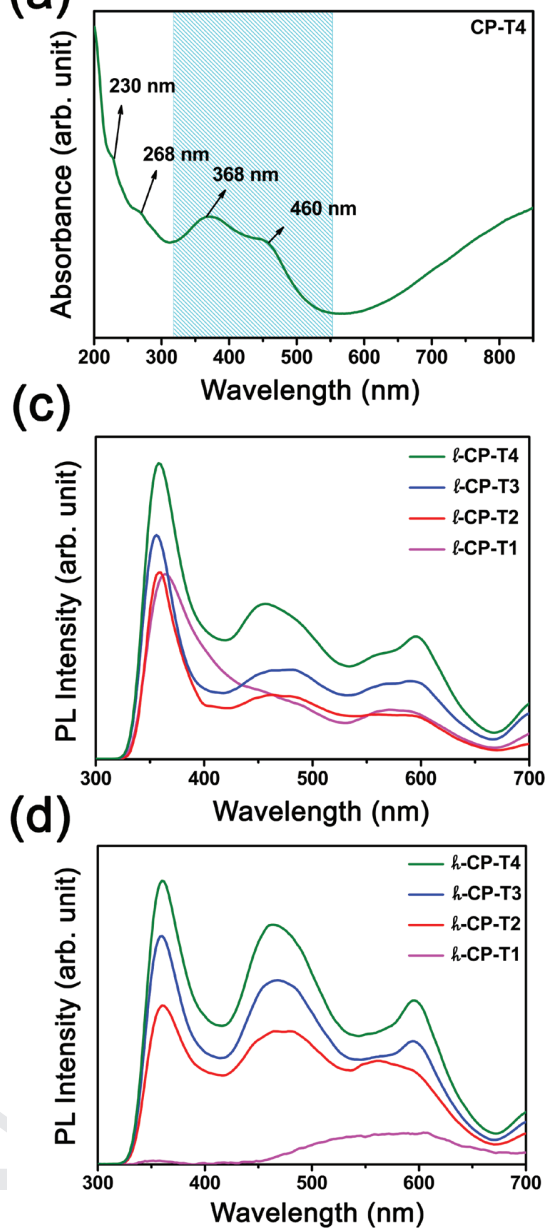

(b)

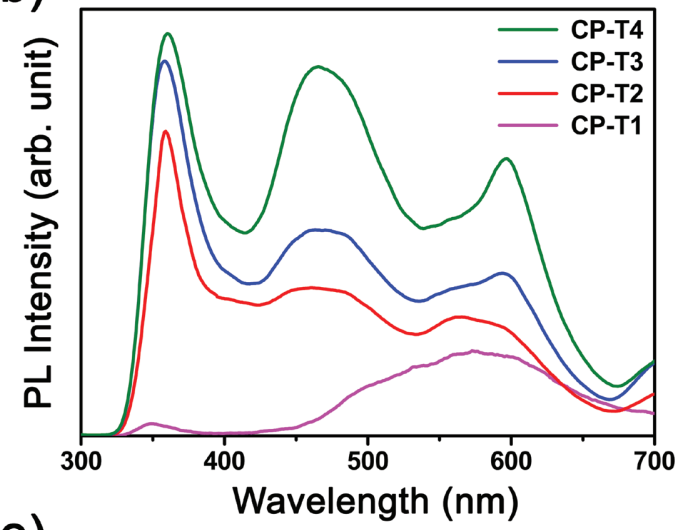

(e)

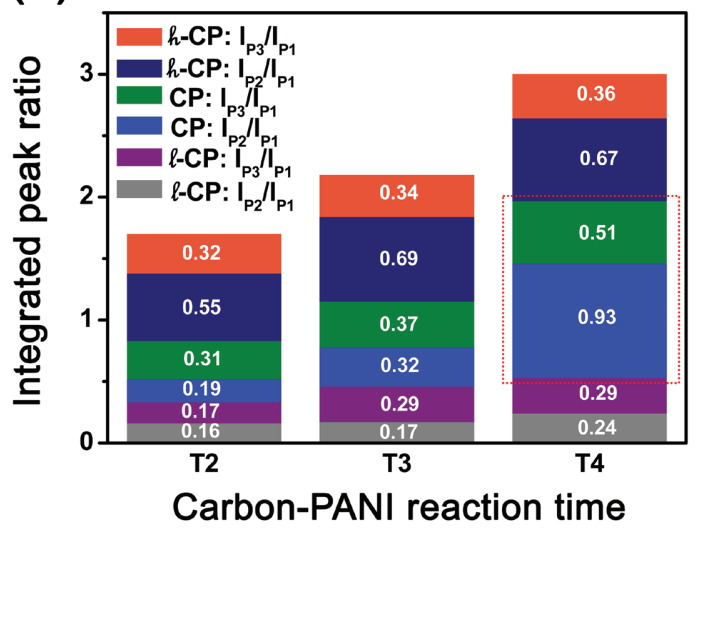

Figure 4. Optical study of CNP incorporated PANI samples. a) UV-vis absorption spectra of a typical sample. b) Triple band PL emission $\left(\lambda_{\mathrm{EM}}=\right.$ 360,466 , and $596 \mathrm{~nm}$ ) of CNP incorporated PANI for sample series of CP in aqueous medium with the excitation of $280 \mathrm{~nm}$. It has been displayed that the PL emission around 466 and $596 \mathrm{~nm}$ comparatively are stronger with increasing functionalization time. Triple band PL emission for sample series of c) I-CP and d) h-CP. e) Plot of integrated areas of $I_{\mathrm{P} 2} / I_{\mathrm{P} 1}$ and $I_{\mathrm{P} 3} / I_{\mathrm{P} 1}$ PL peak for three different series of samples. The red box indicates the most optimised series (CP-T4) resulting in most effective PL emission. 
1 to synergic effect between the two, CNPs and PANI for their 2 conjugation. Another peak at $\approx 596 \mathrm{~nm}\left(I_{\mathrm{P} 3}\right)$ can be assigned as 3 a luminescence effect from functionalized CNPs. Emission at 4 530-570 nm has been previously reported for $\mathrm{NH}_{2}$-functional5 ized carbon materials. ${ }^{[17]}$ In the present work, a further red-shift 6 in PL emission for CNP-PANI should have originated from 7 the change of electronic structure by formation of functional8 ized CNP-PANI. However initially, for time T1 we have noticed 9 a low intense broad emission peak originating from 400 to $10750 \mathrm{~nm}$, may be a combined effect of peak $I_{\mathrm{P} 2}$ and $I_{\mathrm{P} 3}$. The 11 result can be attributed to a weak interaction between CNPs 12 and PANI in the elementary form of reaction. Also, a tiny peak 13 around $350 \mathrm{~nm}$ has been originated due to PANI. But, with 14 increasing polymerization time the three peaks come out and 15 get intensified. This observation implies that the strong triple16 band emission state exists in CNP-PANI hybrid systems, unlike 17 most previously reported PL of carbon particles. ${ }^{[47,48]}$ The com18 parative PL emission for these three consecutive wavelengths 19 has been influenced by the reaction time between CNPs and 20 PANI. The phenomenon can be due to the development of an 21 oxidation-resistant reaction through the introduction of CNPs 22 in aniline monomer during polymerization. This can be corre23 lated with the results of Q/B ring ratio, determined from FTIR 24 analysis. Decreasing nature in Q/B signifies the presence of 25 more reduced emeraldine segments in CNP-PANI composite,

26 can be ascribed to strong $\pi-\pi$ interaction between CNPs and 27 PANI. Consequently, CNPs interact with polymeric chains 28 where the incorporated carbon nanoparticles reduce the PANI 29 through charge transfer, increasing strongly with reaction 30 time. As a result, with time (from T1 to T4) CNPs incorporated 31 within PANI lead to an influential effect on PL emissions for $32 I_{\mathrm{P} 2}$ and $I_{\mathrm{P} 3}$. The contributing effect of PL emission due to func33 tionalized form of CNPs can be analyzed from the integrated 34 peak ratio of $I_{\mathrm{P} 2} / I_{\mathrm{P} 1}$ and $I_{\mathrm{P} 3} / I_{\mathrm{P} 1}$, where $I_{\mathrm{P} 1}$ is the PL emission 35 from PANI itself. Interestingly, the ratios $\left(I_{\mathrm{P} 2} / I_{\mathrm{P} 1}\right.$ and $\left.I_{\mathrm{P} 3} / I_{\mathrm{P} 1}\right)$ 36 rise from $\mathrm{T} 1$ to $\mathrm{T} 4$ indicating strong $\pi-\pi$ interaction within 37 the composite. Peak intensities were recorded as maximum 38 values for the sample CP-T4. All these features are well distin39 guished from other previously reported CNPs. ${ }^{[17]}$ The optical 40 performance relates to the change of chemical structure or local 41 environment of CNPs that have occurred here due to the func42 tionalization of common oven produced carbon material into 43 polyaniline matrix.

44 The above study has gradually revealed how we have 45 achieved interesting PL properties from the CNP-PANI com46 posites which had no such distinguished and sharp features 47 for its individual components whether it is CNPs or PANI (just 48 broad hump-like spectra as shown in Figure S5, Supporting 49 Information). Additionally, we have studied more details in the 50 variation of PL emission correlated with the change in CNPS 51 concentration within PANI. Figure 4c,d shows PL emission 52 spectra for lower concentration of CNPs (named with 1-CP) 53 and higher concentration of CNPs (named with h-CP), respec54 tively. Though for higher CNP concentration (than that in CP 55 series samples) there is no pronounced change in the pattern 56 of emission, but for lower CNP concentration we have noticed 57 some anomalous features (compatible with the previously dis58 cussed reasons). For example, the PL emission spectrum for 59 sample 1-CP-T1 (with low CNP concentration and initial stage of functionalization) exhibits a strong peak of $I_{\mathrm{P} 1}$ at $364 \mathrm{~nm}$ with 1 a weak shoulder of $I_{\mathrm{P} 2}$ unlike other CNP-PANI samples. The 2 initial hump of second PL emission peak $\left(I_{\mathrm{P} 2}\right)$ can be attributed 3 to weak synergic effect between the two, CNPs and PANI for 4 their conjugation. The low intense blunt peak at $\approx 596 \mathrm{~nm}\left(I_{\mathrm{P} 3}\right) \quad 5$ can also be understood as a feeble luminescence effect from the 6 weakly functionalized CNPs. Further, the integrated peak area 7 ratio of $I_{\mathrm{P} 2} / I_{\mathrm{P} 1}$ and $I_{\mathrm{P} 3} / I_{\mathrm{P} 1}$ are getting higher with increasing 8 reaction time, which is justifiable by the previous analysis due 9 to the presence of lower CNP concentration and lower function- 10 alization effect therefore. More explicitly, the integrated areas 11 of as discussed peak ratio $\left(I_{\mathrm{P} 2} / I_{\mathrm{P} 1}\right.$ and $\left.I_{\mathrm{P} 3} / I_{\mathrm{P} 1}\right)$ for all the series 12 of samples (l-CP, CP, and h-CP) for functionalization time 13 T2, T3, and T4 have been illustrated in Figure 4e. According 14 to this analytical data, it is clear that the luminescence effect 15 from CNP-PANI composite is progressive for sample series of 16 $\mathrm{CP}$ and it effectively shows promising results for CP-T4, com- 17 parable with all others photoluminescence effects.

In accordance with the absorption spectra of CNP-PANI composites, we measured PL excitation-emission (PLE) maps within the UV-visible range. Figure 5 shows a consecutive series of PLE maps of samples CP-T1, CP-T2, CP-T3, and CP-T4. An acute arena of PL emission centered at $596 \mathrm{~nm}\left(I_{\mathrm{P} 3}\right)$ clearly reveals in all the PLE maps which is the effect of surface passivation on the optical properties of functionalized CNPs within PANI. All the consecutive PLE maps have been followed by the formation of metamorphosis in arena of $I_{\mathrm{P} 3}$.

The shape of the PL arena has been changed from circular to elliptical with increasing interaction between CNPs and PANI. These observed features of metamorphosis in PL arena are the results of narrower and sharper emission of $I_{\mathrm{P} 3}$ with increasing T-value. Decrease in peak broadening is because of increase in surface passivation with ongoing functionalization time duration, causing less defect states. It can also be noticed that the most intense point of the arena (reddish part of the PLE map) is shifted toward exposition to higher excitation wavelength from T1 to T4. In detail, the demonstration of PL emission at $596 \mathrm{~nm}$ when the excitation wavelength is changed from 420 to $560 \mathrm{~nm}$ has been shown in the Supporting Information. Figure S6 (Supporting Information) shows how the emission peak at $596 \mathrm{~nm}$ first increases for increasing excitation wavelength and reaches a maximum at PLE of $540 \mathrm{~nm}$ and then starts to decrease with further increase in excitation wavelength, while the peak position remains unchanged. Generally, the PL reported for carbon dots are highly excitation-dependent originating from inhomogeneous dot size effect and or surface defects. However, in the present case, PL of CNPs is thoroughly showing excitation-independent behavior which is considered to be related to the surface passivation effect because of using $\mathrm{NH}_{2}$-group-rich aniline as the starting monomer. During the in situ polymerization process to form PANI while simultaneously functionalizing CNPs, it is the surface amino groups that passivate the traps on surface of CNPs and lead to a single transition mode. In addition, another interesting observation in PLE maps has been noticed for all the CNP-PANI composites at the elementary reaction condition (i.e., T1 state). PLE maps of 1-CP-T1 and h-CP-T1 have been given in Figure S7 (Supporting Information). An anomalous behavior of emission has been noticed for 1-CP-T1 where the $I_{\mathrm{P} 1}$ displays more intense

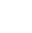
.

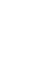
.

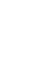
. . . . . . , 

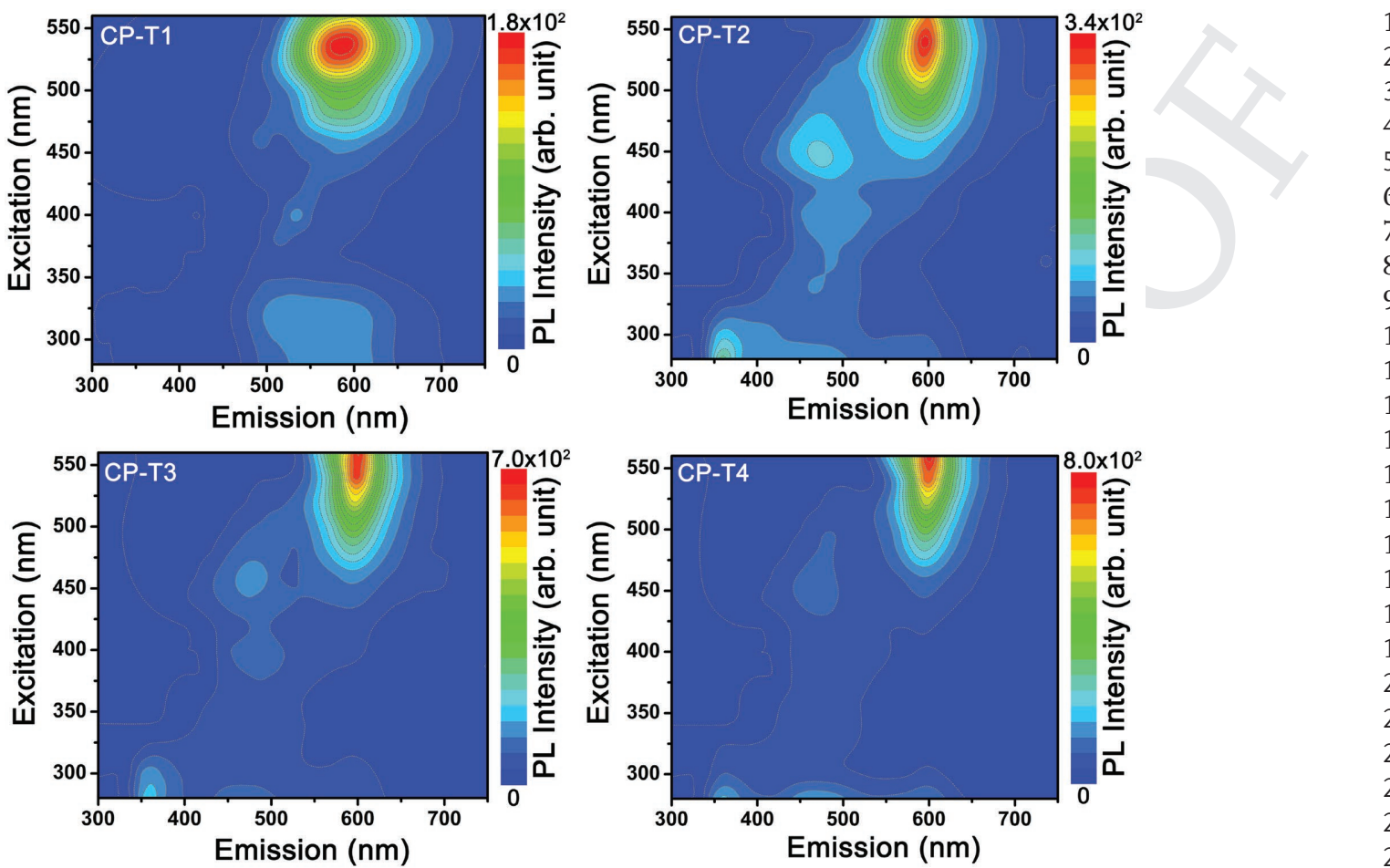

Figure 5. PLE maps of the solution of CP series for different CNP-PANI functionalization times (T1, T2, T3, and T4). An intense arena of the PL emis- 26 sion centered at $596 \mathrm{~nm}\left(I_{P 3}\right)$ has been patterned for all the samples, followed by the metamorphosis in shape with increasing functionalization time, 27 that is, duration of interaction between CNPs and PANI. Other emission centers of $I_{\mathrm{P} 1}$ and $I_{\mathrm{P} 2}$ have also been noticed for CP-T2, CP-T3, and CP-T4, 28 which are comparatively less intense.

emission comparable with $I_{\mathrm{P} 3}$, indicating less functionalization effect of CNPs with PANI.

As-synthesized CNP-PANI samples were also found to be strongly luminescent on a substrate (deposited as thin films). As shown in Figure 6, the fluorescence image of a typical CNPPANI sample obtained by confocal microscopy exhibits a bright and intense luminescence effect from the material (excitation wavelength: $488 \mathrm{~nm}$ ). However, it should be cautioned that the observed brightness variation might also be subject to effects from some measurement issues, such as some luminescent centers being slightly out of the focal plane.

\subsection{Concentration-Dependent PL}

Furthermore, Figure 7a shows a clear correlation of $I_{\mathrm{P} 2} / I_{\mathrm{P} 1}$ and $I_{\mathrm{P} 3} / I_{\mathrm{P} 1}$ with the concentration of a typical sample series of CP-T4, dispersed in the solution. Each time sample concentration was decreased to half of the previous one. The exponential decreasing factor of the integrated peak ration of $I_{\mathrm{P} 2} / I_{\mathrm{P} 1}$ and $I_{\mathrm{P} 3} / I_{\mathrm{P} 1}$ indicates that these changes in the dispersion of the CP-T4 can have large effects on the number of reabsorption events a photon undergoes. It can be because of photon reabsorption $^{[49,50]}$ at the lower wavelength emission (PL emission at $\approx 360 \mathrm{~nm}$ ) which is most strongly affected by concentration of CNP-PANI in the solution, over the total detected emissions. For the range of concentrations investigated, it shows a purely monotonic trend with the peak rations.
Again, we have investigated the PL emission around at 32 $596 \mathrm{~nm}$ with excitation of $500 \mathrm{~nm}$. The PL intensity is com- 33 pletely dependent on the sample concentration. Initially, PL 34 intensity increases with decreasing factor of sample concentra- 35 tion in the solution. But further decrease of sample concentra- 36 tion, PL intensity decreases again. The phenomenon can be 37

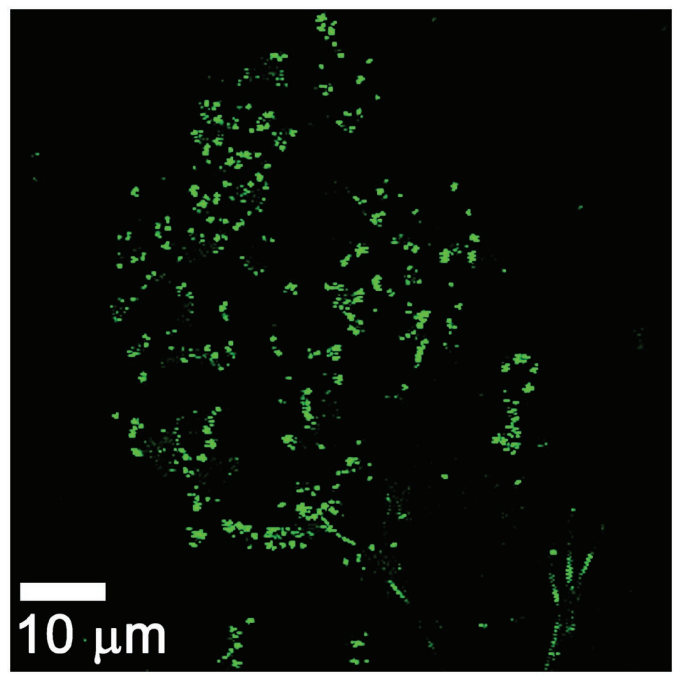

Figure 6. A confocal fluorescence microscopy image of a typical CNP. 57 PANI composite at room temperature (irradiated under the laser excita- 58 tion wavelength of $488 \mathrm{~nm}$ ). 


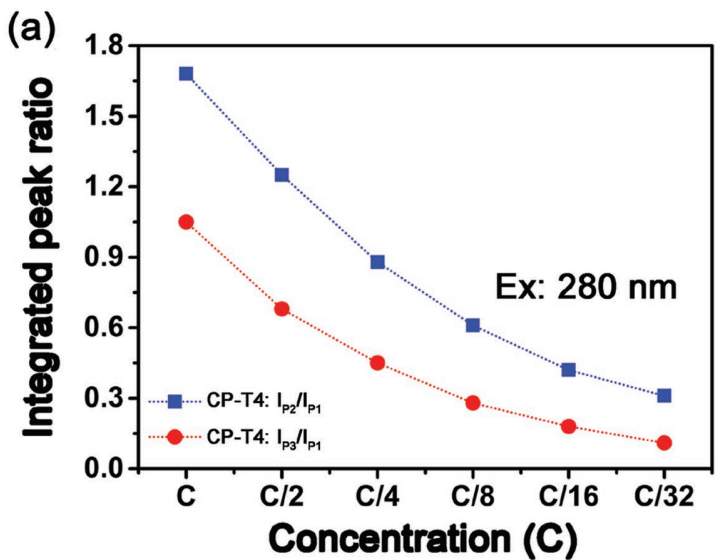

(b)

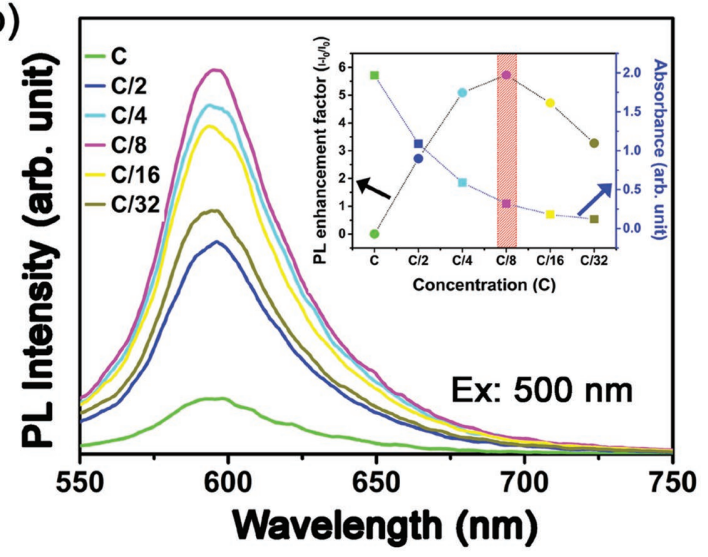

Figure 7. a) The integrated peak ration of $I_{\mathrm{P} 2} / I_{\mathrm{P} 1}$ and $I_{\mathrm{P} 3} / I_{\mathrm{P} 1}$ decreases exponentially with the decreasing factor of CP-T4 sample concentration in the solution for the excitation wavelength of $280 \mathrm{~nm}$. b) The concentration-dependent emission with the excitation of $500 \mathrm{~nm}$, for the sample series of CP-T4. Inset: Plots the variation of PL enhancement factor of CP-T4 dispersion and the UV absorption at the excitation wavelength (i.e., $500 \mathrm{~nm}$ ) for five different sample concentrations. Red line indicates the optimum concentration of the sample for best PL enhancement factor.

attributed to the effect of emission photon scattering ${ }^{[51]}$ within the sample. The emission photon scatters when the sample concentration in the dispersion is higher, resulting lesser PL emission detection. But the effect reduces with decreasing concentration and PL enhancement has been observed. But, PL intensity again decreases after reaching the optimum sample concentration, that is, when the scattering factor minimizing could not affect over the real emission from the samples. Figure $7 \mathrm{~b}$ shows the concentration-dependent emission with the excitation of $500 \mathrm{~nm}$, for the sample series of CP-T4. Inset of Figure $7 \mathrm{~b}$ plots the variation of PL enhancement factor of CP-T4 dispersion with function of UV absorption at the excitation wavelength (i.e., $500 \mathrm{~nm}$ ) for five different sample concentrations. The PL enhancement factor is defined as $\left(I-I_{0}\right) / I_{0}$, where $I_{0}$ and $I$ are the initial and the enhanced PL intensities, respectively. Interestingly, UV absorption at the excitation wavelength decreases monotonously with the decreasing concentration of CP-T4, but the trend is not observed same for the PL enhancement factor. At the concentration of $\mathrm{C} / 8$, though the absorption is lower at the excitation, the emission at $596 \mathrm{~nm}$ is maximum.

\subsection{PL Probing for lon Sensing}

1

Recently, photoluminescent carbon dot-based nanosensors are being extensively developed for practical field application. ${ }^{[52,53]} 4$ So, here we have tried to explore the feasibility of using as- 5 prepared CNP-PANI as a PL sensing platform and found that 6 it can be actually used as an effective PL probe for the detec- 7 tion of $\mathrm{Fe}^{+3}$ ion. It is well-known that $\mathrm{Fe}^{+3}$ ions are indispen- 8 sable for a large number of living systems and act as a key material for many important biochemical processes like oxygen uptake, oxygen metabolism, and electron transfer. So, detection of such metal ions is considered to be highly desirable. Here in Figure 8a, the PL intensity of as-synthesized CNP-PANI (optimized one, i.e., CP-T4; excitation wavelength: $500 \mathrm{~nm}$ ) at $596 \mathrm{~nm}$ was observed to decrease gradually with increasing $\mathrm{Fe}^{+3}$ ion concentration, indicating that $\mathrm{Fe}^{+3}$ can effectively quench the photoluminescence of CNP-PANI.

This quenching can be simply attributed to nonradiative electron-transfer process generated due to adsorption of $\mathrm{Fe}^{+3}$ ion on the surface of material that involves partial transfer for an electron in the excited state to the $d$ orbital of $\mathrm{Fe}^{+3}$. To get insight into the quenching mechanism, corresponding data were analyzed by the familiar Stern-Volmer equation. The full concentration range $\left(0-60 \times 10^{-6} \mathrm{M}\right)$ plot as presented in Figure $8 \mathrm{~b}$ is not rigorously following the conventional linear Stern-Volmer equation inferring that both dynamic and static quenching processes occur in the above-mentioned analytical system. Other two types of CNP-PANI samples (h-CP-T4 and 1-CP-T4) have also responded (Figure S8, Supporting Information) in this sensing experiment but the quenching effect is much lower than that of CP-T4 (see inset of Figure 8b). A PL emission titration experiment was also performed for CP-T4 for evaluating the quantitative detection capability of this system at a signal-to-noise ratio of 3 . The limit of detection (LOD) of $\mathrm{Fe}^{+3}$ is estimated to be about $12 \times 10^{-9} \mathrm{M}$ based on three times standard deviation rule (LOD $=3 \delta /$ slope) from the linear fit of Stern-Volmer equation in the range of $0-160 \times 10^{-9} \mathrm{M}$ (the correlation coefficient $R^{2}$ is 0.99 , Figure S9, Supporting Information). The result is significantly lower compared to the previous results. ${ }^{[54,55]}$ So, the material is highly sensitive toward detecting $\mathrm{Fe}^{+3}$ ions. In addition, to evaluate the detection ability of this system for other various biologically and environmentally relevant metal ions, we investigated the PL quenching effect for ions including $\mathrm{Na}^{+}, \mathrm{K}^{+}, \mathrm{Ca}^{+2}, \mathrm{Cd}^{+2}, \mathrm{Mg}^{+2}$, $\mathrm{Mn}^{+2}, \mathrm{Fe}^{+3}, \mathrm{Ag}^{+}, \mathrm{Cu}^{+2}, \mathrm{Co}^{+2}, \mathrm{Zn}^{+2}$, and $\mathrm{Pb}^{+2}$. The response of the assay was tested for these different ions at a concentration of $10 \times 10^{-6} \mathrm{M}$ under the same conditions as those used with $\mathrm{Fe}^{+3}$ (Figure S10, Supporting Information). Remarkably, no significant PL quenching effect was observed for adding the other metal ions (some showed minimal quenching effect such that the influence is almost negligible) except $\mathrm{Cu}^{+2}$ ions. The $\mathrm{Cu}^{+2}$ ions showed dramatic decrease in the PL intensity; herein the quenching can be ascribed to the fact that $\mathrm{Cu}^{+2}$ is a paramagnetic ion with unfilled $\mathrm{d}$ shell and hence can result in quench of the PL intensity via electron or energy transfer. Though $\mathrm{Cu}^{+2}$ showed a relatively strong quenching effect (Figure S11, Supporting Information) but, while tested for titration experiment for comparing the detection ability with that of $\mathrm{Fe}^{+3}$ under same conditions, the results demonstrated for $\mathrm{Fe}^{+3}$ ions are far better

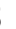
. . . 10 11 12 13 14 15 16
17 17 18 19 20 21 22 23 24 25 26 27 28 29 30 31 32 33 34 35 36 37 38 39 40 41 42 43 44 45 46 47 48 49 50 51 52 53 54 

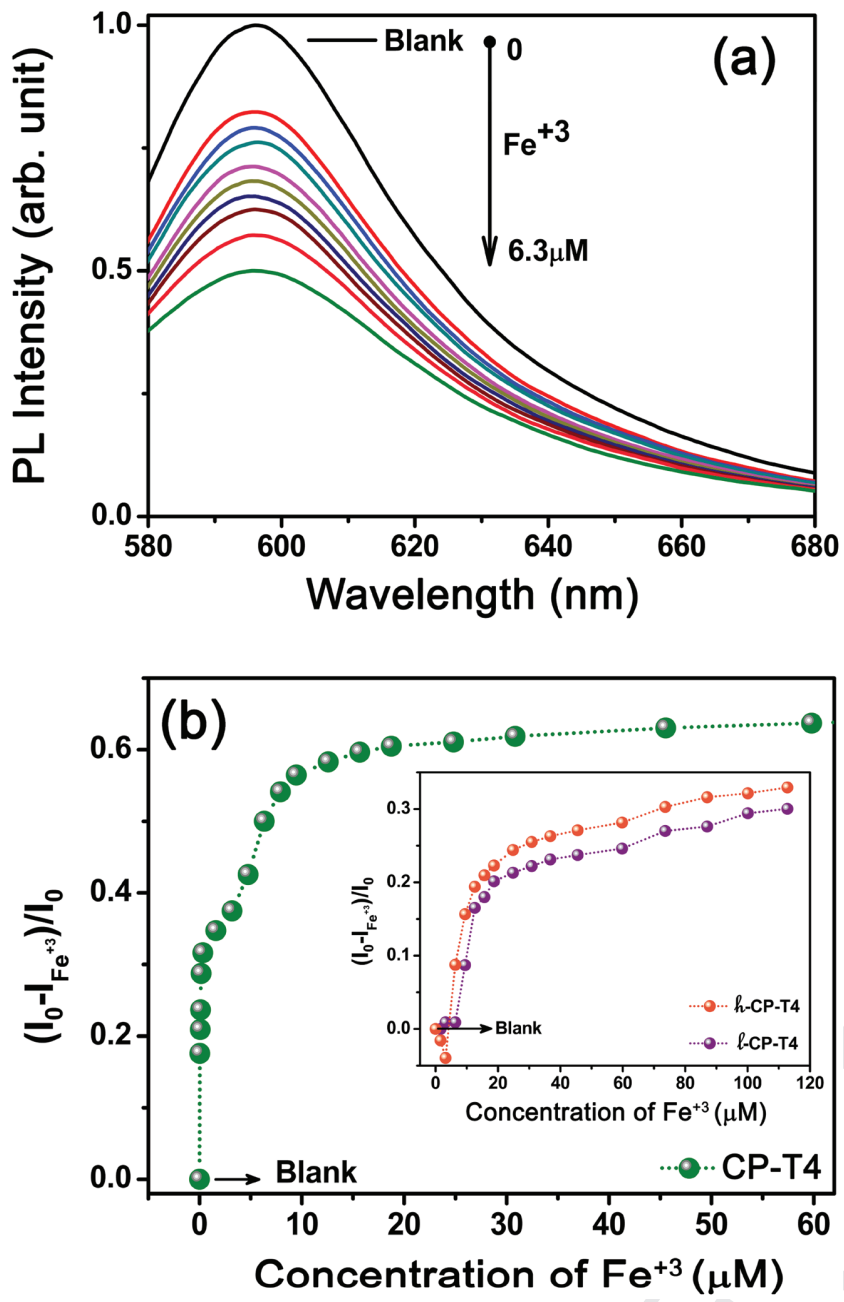

Figure 8. Ion detection by $\mathrm{PL}$ spectra. a) Dependence of $\mathrm{PL}$ emission spectra $\left(\lambda_{\mathrm{EX}}=500 \mathrm{~nm}\right)$ on $\mathrm{Fe}^{+3}$ ions concentration $\left(0-6.3 \times 10^{-6} \mathrm{M}\right)$ in mixture with CP-T4 sample $\left(10 \mu \mathrm{g} \mathrm{mL}^{-1}\right)$. b) Corresponding ion detection plot of relative $\mathrm{PL}$ emission $\left(\left(I_{0}-I_{\mathrm{Fe}}{ }^{+3}\right) / I_{0}\right.$, where $I_{0}$ is the $\mathrm{PL}$ emission of assynthesized material without any ions and $I_{\mathrm{Fe}}{ }^{+3}$ is the $\mathrm{PL}$ emission upon addition of $\mathrm{Fe}^{+3}$ ion) at $596 \mathrm{~nm}$ for $\mathrm{Fe}^{+3}$ concentration $\left(0-60 \times 10^{-6} \mathrm{M}\right)$ for sample CP-T4. Inset: The same detection plot of $\mathrm{Fe}^{+3}$ ions concentration $\left(0-120 \times 10^{-6} \mathrm{M}\right)$ for the sample of $h$-CP-T4 (orange color) and $\ell$-CP-T4 (purple color). It has been noted that the detection value strikes at 0.56 for $9.5 \times 10^{-6} \mathrm{M}$ of $\mathrm{Fe}^{+3}$ ions for CP-T4, but the value is much lower for the sample of $h$-CP-T4 and $\ell$-CP-T4 $(0.09$ and 0.16 , respectively). However, from the linear fitting, the estimated LOD for $\mathrm{Fe}^{+3}$ ion is $\approx 12 \times 10^{-9} \mathrm{M}$ for CP-T4.

and also more interesting. $\mathrm{Fe}^{+3}$ ion is already a well-known effcient fluorescence quencher due to its paramagnetic properties via electron or energy transfer. Here, the $\mathrm{Fe}^{+3}$ could be preferentially binding with nitrogen atom of the amino groups ${ }^{[56]}$ residing in the polymeric backbone of the composite. Therefore, the $\mathrm{Fe}^{+3}$ ions can be adsorbed on the material surface with its higher affinity for the amino group itself, which would facilitate charge transfer further and thus restrain exciton recombination, leading to significant fluorescence quenching. Thus, as-synthesized CNP-PANI showed high detection selectivity for $\mathrm{Fe}^{+3}$ ions among the studied metal ions, except $\mathrm{Cu}^{+2}$ ions. So basically, the strong affinity between the $\mathrm{Fe}^{+3}$ ions and nitrogen atom of the amino groups on CNP-PANI surface enables this 1 composite material as a promising nanosensing platform for 2 $\mathrm{Fe}^{+3}$ ions with high efficiency and selectivity criteria. Even if 3 $\mathrm{Cu}^{+2}$ at high concentration interferes with $\mathrm{Fe}^{+3}$ detection, the 4 issue can be circumvented by using ethylenediaminetetraacetic 5 acid (EDTA) as a strong chelating agent of $\mathrm{Cu}^{+2}$ ions, ${ }^{[57]}$ indi- 6 cating also the ability of transforming our system toward the 7 $\mathrm{Fe}^{+3}$ selectivity.

In summary, incorporation of carbon nanoparticles within 9 polyaniline has been proven to be an effective strategy for pro- 10 ducing fluorescent CNPs. Moreover, no further chemical modi- 11 fication is needed before using cooking oven produced trash 12 carbon materials; thus, it incorporates green nanotechnology 13 for efficient reduction of waste products and offers the advan- 14 tages of an economic and less toxic procedure for application. 15 Such CNP-PANI nanocomposites exhibited excitation-wave- 16 length-independent photoluminescent behavior with lumi- 17 nescence intensity progressively increasing as the CNPs con- 18 tent increased; the optimal condition was also achieved where 19 photoluminescence reached its maximum. The CNP-PANI 20 nanocomposites exhibit broad PL spectra covering most of the 21 visible range but with three distinct intense peaks, thus opening 22 up the possibility of using these materials as luminophores 23 in white light LEDs and multicolor patterning systems. As- 24 synthesized CNP-PANI has been further tested as a sensing 25 platform for metal ions which provided data in support of its 26 highly sensitive detection ability for $\mathrm{Fe}^{+3}$ and $\mathrm{Cu}^{+2}$ ions. Our 27 present study is important because it provides a simple, low- 28 cost route toward incorporating carbon nanoparticles within a 29 polymer to get CNP-PANI that can be used further for sensing 30 and other optoelectronics devices. 31

\section{Experimental Section}

Reagents and Materials: CNPs used in the present work were simply the waste carbon product collected from cooking oven (the morphology of the waste carbon comprised of CNPs is discussed tater in the Results and Discussions). Aniline monomer (Aldrich) was distilled under vacuum prior to use. Ammonium persulfate (APS; 99.99\%, Aldrich) and all other reagents like hydrochloric acid $(\mathrm{HCl} ; 35 \%$, Alfa Aesar) and absolute ethanol (>99.99\%, Alfa Aesar) were used as received without further purification. The salts used for ion detection are $\mathrm{NaCl}, \mathrm{KCl}, \mathrm{CaCl}_{2}$, $\mathrm{CdCl}_{2}, \mathrm{MgCl}_{2}, \mathrm{MnCl}_{2}, \mathrm{FeCl}_{3}, \mathrm{AgNO}_{3}, \mathrm{CuCl}_{2} .2 \mathrm{H}_{2} \mathrm{O}, \mathrm{CoCl}_{2}, \mathrm{PbCl}_{2}$, and $\mathrm{ZnCl}_{2}$. Deionized (DI) water was used throughout all the experiments.

Preparation of CNP-PANI: Waste carbon products were used here without any further chemical modification or purification. The preparatory strategy was both green and economic. The polymerization was done in 1:1 (v/v) ethanol-water media. Ethanol here helped to disperse the CNPs within precursor during reaction and also slow down the polymerization process to some extent. The CNPs were introduced into the polymer following a typical chemical oxidative polymerization of aniline at $0{ }^{\circ} \mathrm{C}$ (ice bath) in presence of APS as oxidant and $\mathrm{HCl}$ as dopant. Aniline was added to $20 \mathrm{~mL}$ of ethanol-water solution and cooled at $0{ }^{\circ} \mathrm{C}$. Some amount of CNPs (for different samples preparation, $1.1,2.2$, and $4.4 \mathrm{mg}$ ) was dispersed in $10 \mathrm{~mL}$ of ethanolwater mixture (to ensure stable dispersion it was mildly stirred for $1 \mathrm{~h}$ on a magnetic stirrer with $430 \mathrm{rpm}$ speed) and then APS and $\mathrm{HCl}$ (both used with the same number of moles as the number of moles of aniline monomer) were added to it. After that, this mixture was kept for cooling at $0{ }^{\circ} \mathrm{C}$ for few minutes and then was dropwise added to the precooled monomer solution with $10 \mathrm{~s}$ shaking. The polymerization was allowed to continue unagitated for $1.5 \mathrm{~h}$ or more (for other samples with time 
variations, e.g., 2, 2.5, and $3 \mathrm{~h}$ ). The dark green precipitate was collected after filtration and several times washing with DI water and methanol and was finally dried in a vacuum oven at $60^{\circ} \mathrm{C}$ for $24 \mathrm{~h}$. Either powders or films were obtained depending on the nature of characterization requirements. The optimized sample was obtained by using $2.2 \mathrm{mg}$ of CNPs; hence the following nomenclature is used: CP for the series of the optimized ones with different time durations like T1 (1.5 h), T2 (2 h), T3 $(2.5 \mathrm{~h}), \mathrm{T} 4(3 \mathrm{~h})$; l-CP and $h$-CP for lower and higher concentrations of CNPs (i.e., 1.1 and $4.4 \mathrm{mg}$ ), respectively.

Characterizations: The measurement of phase imaging under bias voltage has been performed by AFM (Asylum MFP-3D) in tapping mode using high-resolution conducting probe. Morphological studies and composition analysis were carried out by FESEM-FIB (Carl Zeiss Auriga Crossbeam microscope) equipped with an EDX (OXFORD) analyser. The samples were further characterized by FTIR spectroscopy and UV-vis-NIR spectroscopy (Shimadzu, UV-3101PC). FTIR spectra for the samples were acquired using an attenuated total reflectance (ATR) sampling accessory (Smart iTR) equipped with a single-bounce diamond crystal on a Thermo Nicolet 6700 Spectrometer (used in the absorbance mode at 50 scans with a resolution of $4 \mathrm{~cm}^{-1}$ for frequency range $400-4000 \mathrm{~cm}^{-1}$ ). XPS was performed on a KRATOS Analytical AMICUS instrument equipped with an achromatic Al $\mathrm{K} \alpha \mathrm{X}$-ray source $(1468.6 \mathrm{eV})$. For a typical analysis, the source was operated at voltage of $10 \mathrm{kV}$ and current of $10 \mathrm{~mA}$. The survey spectra were acquired using a step of $1 \mathrm{eV}$, whereas $0.1 \mathrm{eV}$ was employed for acquiring narrow scan regions. The pressure in analysis chamber was less than $5 \times 10^{-6}$ $\mathrm{Pa}$. The deconvolution of peaks was done by fitting with a GaussianLorentzian ratio of 0.5 and a constant FWHM of $1.9 \mathrm{eV}$. The binding energy of all samples was corrected for eventual charging by shifting the convoluted peak intensity of $\mathrm{Cls}$ to $284.6 \mathrm{eV}$. The Raman measurements with the excitation laser line of $532 \mathrm{~nm}$ were performed using a WITEC Alpha300AR+ Confocal Raman system in air ambient environment. The room-temperature PL experiments were performed with a PerkinElmer LS-55 instrument using a Xenon lamp as excitation source with an excitation wavelength range from 280 to $560 \mathrm{~nm}$. Also, the optical image was collected by a confocal laser scanning microscope (LSM 700/Carl Zeiss), with $488 \mathrm{~nm}$ laser. Afterward, the image analysis was performed using ZEN 2.1 software (Carl Zeiss).

Detection of lons: The detection of $\mathrm{Fe}^{+3}$ ions was performed in PBS $\left(10 \times 10^{-3} \mathrm{M}, \mathrm{pH} 7.1\right)$ buffer solution. In a typical run, CNP-PANI sample dispersion (loading $10 \mu \mathrm{g} \mathrm{mL}^{-1}$ ) was added into $3 \mathrm{~mL}$ of PBS buffer in a spectrophotometer quartz cuvette, followed by addition of different concentrations of $\mathrm{Fe}^{+3}$ ions. The PL spectra were recorded after reaction for $15 \mathrm{~min}$. To investigate the selectivity of $\mathrm{Fe}^{+3}$ ions and possible interference of other cations, following salts were used: $\mathrm{NaCl}, \mathrm{KCl}$, $\mathrm{CaCl}_{2}, \mathrm{CdCl}_{2}, \mathrm{MgCl}_{2}, \mathrm{MnCl}_{2}, \mathrm{FeCl}_{3}, \mathrm{AgNO}_{3}, \mathrm{CuCl}_{2} \cdot 2 \mathrm{H}_{2} \mathrm{O}, \mathrm{CoCl}_{2}, \mathrm{FeSO}_{4}$, and $\mathrm{ZnCl}_{2}$, and tested with the sample stock solution $\left(10 \times 10^{-6} \mathrm{M}\right)$ in buffer in a similar way. All the experiments were performed at room temperature.

\section{Supporting Information}

Supporting Information is available from the Wiley Online Library or from the author.

\section{Acknowledgements}

This work was partially financed by FEDER funds through the COMPETE 2020 Programme and National Funds through FCT under the project UID/CTM/50025/2013 and EXCL/CTM-NAN/0201/2012. S.N. was supported by FCT-MEC under fellowship SFRH/BPD/70367/2010. P.C. acknowledges funding from KAUST.

\section{Conflict of Interest}

The authors declare no conflict of interest.

\section{Keywords}

carbon nanoparticles, functionalization, ion sensing, photoluminescence, polyaniline

Received: September 28, 2017

Revised: October 28, $2017 \quad 6$

Published online:

1] J. Briscoe, A. Marinovic, M. Sevilla, S. Dunn, M. Titirici, Angew. Chem., Int. Ed. 2015, 54, 4463.

[2] X. Guo, C. F. Wang, Z. Y. Yu, L. Chen, S. Chen, Chem. Commun. 2012, 48, 2692.

[3] P. Mirtchev, E. J. Henderson, N. Soheilnia, C. M. Yip, G. A. Ozin, J. Mater. Chem. 2012, 22, 1265

[4] S. Chandra, P. Patra, S. H. Pathan, S. Roy, S. Mitra, A. Layek, R. Bhar, P. Pramanik, A. Goswami, J. Mater. Chem. B 2013, 1, 2375.

[5] H. T. Li, X. D. He, Z. H. Kang, H. Huang, Y. Liu, J. Liu, S. Lian, C. H. T. Sang, X. Yang, S. T. Lee, Angew Chem., Int. Ed. 2010, 49, 4430.

[6] L. Cao, X. Wang, M. J. Meziani, F. Lu, H. Wang, P. G. Luo, Y. Lin, B. A. Harruff, L. M. Veca, D. Murray, S.-Y. Xie, Y.-P. Sun, J. Am. Chem. Soc. 2007, 129, 11318.

[7] S. T. Yang, L. Cao, P. G. Luo, F. Lu, X. Wang, H. Wang, M. J. Meziani, Y. Liu, G. Qi, Y.-P. Sun, J. Am. Chem. Soc. 2009, 131, 11308.

[8] S. J. Zhu, J. H. Zhang, L. Wang, Y. B. Song, G. Y. Zhang, H. Y. Wang, B. Yang, Chem. Commun. 2012, 48, 10889.

[9] B. Kong, A. Zhu, C. Q. Ding, X. M. Zhao, B. Li, Y. Tian, Adv. Mater. 2012, 24, 5844.

[10] S. Liu, J. Tian, L. Wang, Y. Zhang, X. Qin, Y. Luo, A. M. Asiri, 30 A. O. Al-Youbi, X. Sun, Adv. Mater. 2012, 24, 2037.

[11] J. Zhang, Y. Yuan, Z. L. Yu, A. Yu, S. H. Yu, Small 2014, 10, 3662.

[12] X. Li, S. Zhang, S. A. Kulinich, Y. Liu, H. Zeng, Sci. Rep. 2014, 4, 4976.

[13] J. Ju, W. Chen, Biosens. Bioelectron. 2014, 58, 219.

[14] J. Ju, R. Zhang, S. He, W. Chen, RSC Adv. 2014, 4, 52583.

[15] J. Ju, W. Chen, Anal. Chem. 2015, 87, 1903.

[16] J. Ju, R. Zhang, W. Chen, Sens. Actuators, B 2016, 228, 66.

[17] S. Zhu, Y. Song, X. Zhao, J. Shao, J. Zhang, B. Yang, Nano Res. 2015, 8, 355

[18] Z. C. Yang, M. Wang, A. M. Yong, S. Y. Wong, X. H. Zhang, H. Tan, A. Y. Chang, X. Li, J. Wang, Chem. Commun. 2011, 47, 11615.

[19] J. Zhou, X. Zhou, R. Li, X. Sun, Z. Ding, J. Cutler, T. K. Sham, Chem. Phys. Lett. 2009, 474, 320.

[20] S. Qu, H. Chen, X. Zheng, J. Cao, X. Liu, Nanoscale 2013, 5, 5514.

[21] Z. Gan, X. Wu, Y. Hao, Cryst. Eng. Commun. 2014, 16, 4981.

[22] Z. Gan, S. Xiong, X. Wu, T. Xu, X. Zhu, X. Gan, J. Guo, J. Shen, L. Sun, P. K. Chu, Adv. Opt. Mater. 2013, 1, 926.

[23] N. S. Sariciftci, L. Smilowitz, A. J. Heeger, F. Wudi, Science 1992, 258, 1474.

[24] S. Goswami, S. Nandy, T. R. Calmeiro, R. Igreja, R. Martins, E. Fortunato, Sci. Rep. 2016, 6, 19514.

25] Y Liao, C. Zhang, Y. Zhang, V. Strong I. Tang X.-G. Li K. Kalantar-zadeh, E. M. V. Hoek, K. L. Wang, R. B. Kaner, Nano Lett. 2011, 11, 954.

[26] I. Kovalenko, D. G. Bucknall, G. Yushin, Adv. Funct. Mater. 2010, 20, 3979.

[27] Z. Gan, H. Xu, Y. Hao, Nanoscale 2016, 8, 7794.

[28] Y. Dong, R. Wang, H. Li, J. Shao, Y. Chi, X. Lin, G. Chen, Carbon 2012, 50, 2810.

[29] P. Anilkumar, X. Wang, L. Cao, S. Sahu, J.-H. Liu, P. Wang, K. Korch, 58 K. N. TackettII, A. Parenzana, Y.-P. Sun, Nanoscale 2011, 3, 2023.
1 2 3 4 , 7 
[30] S. Zhu, J. Zhang, S. Tang, C. Qiao, L. Wang, H. Wang, X. Liu, B. Li, Y. Li, W. Yu, X. Wang, H. Sun, B. Yang, Adv. Funct. Mater. 2012, 22, 4732.

[31] X. Zhai, P. Zhang, C. Liu, T. Bai, W. Li, L. Daic, W. Liu, Chem. Commun. 2012, 48, 7955 .

[32] S. Nandy, G. Gonçalves, J. V. Pinto, T. Busani, V. Figueiredo, L. Pereira, R. F. P. Martins, E. Fortunato, Nanoscale 2013, 5, 11699.

[33] S. Golczak, A. Kanciurzewska, M. Fahlman, K. Langer, J. J. Langer, Solid State lonics 2008, 179, 2234.

[34] B. P. Payne, M. C. Biesinger, N. S. Mclntyre, J. Electron Spectrosc. Relat. Phenom. 2011, 184, 29.

[35] G. Wu, L. Li, J. H. Li, B. Q. Xu, Carbon 2005, 43, 2579.

[36] Y. Dong, H. Pang, H. B. Yang, C. Guo, J. Shao, Y. Chi, C. M. Li, T. Yu, Angew. Chem., Int. Ed. 2013, 52, 7800.

[37] M. Trchová, J. Stejskal, Pure Appl. Chem. 2011, 83, 1803.

[38] S. Saravanan, C. J. Mathai, M. R. Anantharaman, S. Venkatachalam, P. V. Prabhakaran, Phys. Chem. Solids 2006, 67, 1496.

[39] S. Goswami, U. N. Maiti, S. Maiti, S. Nandy, M. K. Mitra, K. K. Chattopadhyay, Carbon 2011, 49, 2245.

[40] S. G. Bachhav, D. R. Patil, Am. J. Mater. Sci. 2015, 5, 90.

[41] M. C. Bernard, A. Hugot-Le Goff, Electrochim. Acta 2006, 52, 595.

[42] R. V. Salvatierra, M. M. Oliveira, A. J. G. Zarbin, Chem. Mater. 2010, 22, 5222.

[43] R. Zhang, W. Chen, Biosens. Bioelectron. 2014, 55, 83.
[44] C.-L. Li, C.-C. Huang, A. P. Periasamy, P. Roy, W.-C. Wu, C.-L. Hsu, 1 H.-T. Chang, RSC Adv. 2015, 5, 2285.

[45] L. Dennany, P. C. Innis, S. T. McGovern, G. G. Wallace, R. J. Forster, 3 Phys. Chem. Chem. Phys. 2011, 13, 3303.

[46] H. Zengin, B. Erkan, Polym. Adv. Technol. 2010, 21216.

[47] F. Wang, Z. Xie, H. Zhang, C.-Y. Liu, Y.-G. Zhang, Adv. Funct. Mater. 5 2011, 21, 1027.

[48] C. Wang, H. Lin, Z. Xu, Y. Huang, M. G. Humphrey, C. Zhang, ACS 7 Appl. Mater. Interfaces 2016, 8, 6621.

[49] Z. Gan, H. Xu, Y. Fu, J. Phys. Chem. C 2016, 120, 29432.

[50] T. Trupkea, J. Appl. Phys. 2006, 100, 063531. 620.

. Cao, C. Du, Z. Zhuang, W. Chen, J. Mater. Chem. C 2016, 4, 6927

[53] X. Gao, Y. Lu, R. Zhang, S. He, J. Ju, M. Liu, L. Li, W. Chen, J. Mater. 14 Chem. C 2015, 3, 2302.

[54] K. Qu, J. Wang I. Ren, X. Qu, Chem. Eur J. 2013, 19, 7243.

[55] S. Liu, R. Liu, X. Xing, C. Yang, Y. Xu, D. Wu, RSC Adv. 2016, 6, 17 31884

[56] J. Li, Q. Wang, Z. Guo, H. Ma, Y. Zhang, B. Wang, D. Bin, Q. Wei, 19 Sci. Rep. 2016, 6, 23558.

[57] S. Liu, J. Tian, L. Wang, Y. Zhang, X. Qin, Y. Luo, A. M. Asiri, 21 A. O. Al-Youbi, X. Sun, Adv. Mater. 2012, 24, 2037. 
Query

Q1: Please provide TOC keyword.

Q2: Please provide the highest academic title (either Dr. or Prof.) for all authors, where applicable.

Q3: Please define all acronyms at their first appearance in the text.

\section{Q1: TOC Keyword}

Green Nanotechnology

Q2: Highest academic title for all authors, where applicable.

Dr. S. Goswami, Dr. S. Nandy, Dr. J. Deuermeier, Dr. D. Nunes, Dr. S. P. Patole, Prof. P. M. F. J. Costa, Prof. R. Martins, Prof. E. fortunato.

Q3. All acronyms are defined at their first appearance in the text.

Comments: In Acknowledgment part, we have inserted two more funding agencies which are crucial, Project 1D-NEON (GA No. 685758), BET-EU (GA No. 692373); Please insert them.

Also, in the text, we have commented that "l" and "h" should be used in the similar font as they are used in the Figure 4 or 8 and also in the figure captions. 


\section{Reprint Order Form}

E-mail: advsustainsys@wiley-vch.de

Wiley-VCH Verlag GmbH \& Co. KGaA

Advanced Sustainable Systems

Boschstr. 12

69469 Weinheim

Germany

Charges for Reprints in Euro (excl. VAT), prices are subject to change. Minimum order 50 copies; single issues for authors at a reduced price.

\begin{tabular}{l|rrrrrr} 
No. of pages & $\begin{array}{c}\mathbf{5 0} \\
\text { copies }\end{array}$ & $\begin{array}{c}\mathbf{1 0 0} \\
\text { copies }\end{array}$ & $\begin{array}{c}\mathbf{1 5 0} \\
\text { copies }\end{array}$ & $\begin{array}{c}\mathbf{2 0 0} \\
\text { copies }\end{array}$ & $\begin{array}{c}\mathbf{3 0 0} \\
\text { copies }\end{array}$ & $\begin{array}{c}\mathbf{5 0 0} \\
\text { copies }\end{array}$ \\
\hline $\mathbf{1 - 4}$ & $345,-$ & $395,-$ & $425,-$ & $445,-$ & $548,-$ & $752,-$ \\
$\mathbf{5 - 8}$ & $490,-$ & $573,-$ & $608,-$ & $636,-$ & $784,-$ & $1077,-$ \\
$\mathbf{9 - 1 2}$ & $640,-$ & $739,-$ & $786,-$ & $824,-$ & $1016,-$ & $1396,-$ \\
$\mathbf{1 3 - 1 6}$ & $780,-$ & $900,-$ & $958,-$ & $1004,-$ & $1237,-$ & $1701,-$ \\
$\mathbf{1 7 - 2 0}$ & $930,-$ & $1070,-$ & $1138,-$ & $1196,-$ & $1489,-$ & $2022,-$ \\
\hline every additional & $147,-$ & $169,-$ & $175,-$ & $188,-$ & $231,-$ & $315,-$ \\
4 pages & & & & & &
\end{tabular}

\section{Please send me and bill me for}

$\square$ no. of reprints $\square$

airmail (+25 Euro)

surface mail

Fedex No.:

$\square$ no. of issue

(1 copy: 54 Euro)

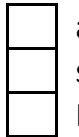

airmail (+ 25 Euro)

surface mail

Fedex No.:

high-resolution PDF file (330 Euro)

E-mail address:

Special Offer:

If you order 200 or more reprints you will get a PDF file for half price.

Please note: It is not permitted to present the PDF file on the internet or on company homepages.

Cover Posters (prices excl. VAT)

Posters of published covers are available in two sizes:

DIN A2 $42 \times 60 \mathrm{~cm} / 17 \times 24$ in (one copy: 39 Euro)

DIN A1 $60 \times 84$ cm / 24 x 33in (one copy: 49 Euro) Postage for shipping posters overseas by airmail:

\section{+25 Euro}

Postage for shipping posters within Europe by surface mail: + 15 Euro

Date, Signature
Manuscript No.:

Customer No.: (if available)

Purchase Order No.:

Author:

Date:

Information regarding VAT: Please note that from German sales tax point of view, the charge for Reprints, Issues or Posters is considered as "supply of goods" and therefore, in general, such delivery is a subject to German sales tax. However, this regulation has no impact on customers located outside of the European Union. Deliveries to customers outside the Community are automatically tax-exempt. Deliveries within the Community to institutional customers outside of Germany are exempted from the German tax (VAT) only if the customer provides the supplier with his/her VAT number. The VAT number (value added tax identification number) is a tax registration number used in the countries of the European Union to identify corporate entities doing business there. Starting with a country code (e.g. FR for France), followed by numbers.
Send bill to:

I will pay by bank transfer

I will pay by credit card

\section{VISA, Mastercard and AMERICAN EXPRESS}

For your security please use this link (Credit Card Token Generator) to create a secure code Credit Card Token and include this number in the form instead of the credit card data. Click here:

https://www.wiley-vch.de/editorial production/index.php

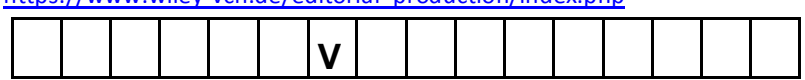

CREDIT CARD TOKEN NUMBER 